\title{
Polioencefalomalacia experimental induzida por amprólio em ovinos ${ }^{1}$
}

\author{
Fabiano J.F. de Sant'Ana², Ana Paula A. Nogueira ${ }^{3}$, Roosevelt I.C. Souza ${ }^{3}$, \\ Suzamar G. Cardinal ${ }^{3}$, Ricardo A.A. Lemos ${ }^{4}$ e Claudio S.L. Barros ${ }^{5 *}$
}

\begin{abstract}
Sant'Ana F.J.F., Nogueira A.P.A., Souza R.I.C., Cardinal S.G., Lemos R.A.A. \& Barros C.S.L. 2009. [Experimentally amprolium-induced polioencephalomalacia in sheep.] Polioencefalomalacia experimental induzida por amprólio em ovinos. Pesquisa Veterinária Brasileira 29(9):747-752. Departamento de Patologia, Universidade Federal de Santa Maria, 97105-900, Santa Maria, RS, Brazil. E-mail: claudioslbarros@uol.com.br

In order to establish an experimental model for the study of the etiology, pathology, and pathogenesis of polioencephalomalacia in ruminants, the condition was induced in five sheep by oral administration of amprolium at daily doses of 500 and $1,000 \mathrm{mg}$ per $\mathrm{kg}$ of body weight respectively for 28-59 days and for 13-39 days. All sheep died or were euthanized in extremis after illness of 3-7 days. Clinical signs included depression, incoordination, midriasis, grinding of the teeth, blindness, and laying down with opisthotonus and paddling movements. Drooling and a sawhorse stance were observed in one sheep and myoclonus in another one. Main gross lesions were restricted to the central nervous system and included swelling of the brain with flattening of telencephalic gyri, and hemorrhages in the parietal and occipital lobes of the telencephalon, in the submeningeal areas of the spinal cord and in the mesencephalon. Histologically, there was segmental laminar neuronal necrosis (red neurons) associated with edema, swelling of endothelial cells, hemorrhages and infiltration by foamy macrophages (gitter cells). These changes were more marked in the frontal, parietal and occipital telecephalic lobes and there was sharp demarcation between the lesions and the adjacent normal neuropile. Additionally, similar, but less marked lesions were seen in the mesencephalon, thalamus and hippocampus. Considering the consistent reproducible aspects of polioencephalomalacia in sheep using amprolium, this may be an useful model for the study of the disease.
\end{abstract}

INDEX TERMS: Diseases of sheep, neuropathology, amprolium, polioencephalomalacia, experimental model.

\footnotetext{
${ }^{1}$ Recebido em 24 de abril de 2009.

Aceito para publicação em 26 de maio de 2009.

Parte da tese de Doutorado do primeiro autor. Trabalho financiado pela Fundação de Apoio ao Desenvolvimento do Ensino, Ciência e Tecnologia do Estado de Mato Grosso do Sul (Fundect), Edital Universal 07/2007.

${ }^{2}$ Programa de Pós-Graduação em Medicina Veterinária, área de concentração em Patologia Veterinária, Centro de Ciências Rurais, Universidade Federal de Santa Maria (UFSM), Santa Maria, RS 97105-900, Brasil. Bolsista do CNPq.

${ }^{3}$ Programa de Pós-Graduação em Ciência Animal, Faculdade de Medicina Veterinária e Zootecnia (FAMEZ), Universidade Federal de Mato Grosso do Sul (UFMS), Av. Filinto Müller s/n, Cidade Universitária, Campo Grande, MS 79070-900, Brasil.

${ }^{4}$ Depto Medicina Veterinária, FAMEZ, UFMS, Campo Grande, MS.

${ }^{5}$ Departamento de Patologia, UFSM, Santa Maria, RS. Pesquisador 1-A do CNPq (Proc.307876/2006-3). *Autor para correspondência: claudioslbarros@uol.com.br
}

RESUMO.- Para estabelecer um modelo experimental para o estudo da etiologia, patologia e patogênese da polioencefalomalacia em ruminantes, a condição foi induzida em cinco ovinos pela administração oral de amprólio nas doses diárias de 500 e $1.000 \mathrm{mg} / \mathrm{kg}$ de peso animal, respectivamente por 28-59 dias e 13-39 dias. Todos os ovinos morreram ou foram eutanasiados in extremis após um curso clínico de 3-7 dias. Os sinais clínicos incluíam depressão, incoordenação, midríase, bruxismo, cegueira e decúbito com opistótono e movimentos de pedalagem. Salivação excessiva e posição de cavalete foi observada em um ovino e mioclonias em um outro. Os principais achados de necropsia restringiam-se ao sistema nervoso central e incluíam tumefação do encéfalo com achatamento dos giros telencefálicos e hemorragias nos lobos parietal 
e occipital do telencéfalo; as hemorragias ocorriam também nas áreas submeníngeas da medula espinhal e do mesencéfalo. Histologicamente, havia necrose segmentar laminar de neurônios (neurônios vermelhos) associada a edema, tumefação de células endoteliais, hemorragias e infiltração por macrófagos espumosos (células gitter). Essas alterações eram mais marcadas nos lobos frontal, parietal e occipital do telencéfalo e havia uma demarcação abrupta entre as lesões e o neurópilo normal adjacente. Adicionalmente, lesões semelhantes, mas menos acentuadas, eram observadas no mesencéfalo, tálamo e hipocampo. Levando em consideração a reproducibilidade regular dos aspectos da polioencefalomalacia em ovinos pela administração de amprólio, esse modelo pode ser útil para o estudo da doença.

TERMOS DE INDEXAÇÃO: Doenças de ovinos, neuropatologia, amprólio, polioencefalomalacia, modelo experimental.

\section{INTRODUÇÃO}

Polioencefalomalacia (PEM) é um diagnostico morfológico para necrose com amolecimento (malacia) da substância cinzenta (polio) do encéfalo. A etiologia e patogênse da PEM em ruminantes ainda é controversa. Embora a PEM de ruminantes tenha sido atribuída inicialmente à deficiência de tiamina (Jensen et al. 1956), foi posteriormente observado que a condição pode ter outras causas, incluindo intoxicação por enxofre (Gould 2000), intoxicação por sal associada à privação de água (Lindley 1977), intoxicação por chumbo (Lemos et al. 2004), administração de determinados anti-helmínticos, como levamisole e tiabendazole (Linklater et al. 1977), administração de análogos da tiamina, como o amprólio (Markson et al. 1974), ingestão de melaço - provavelmente associada à intoxicação por enxofre - (Mella et al. 1976), mudança brusca de pastos ruins para outros de ótima qualidade (Moro et al. 1994), ingestão de plantas ricas em tiaminases (Ramos et al. 2005), intoxicação por Phalaris spp. (Anderton et al. 1994) e infecção por herpesvírus bovino (Carrillo et al. 1983). Em algumas dessas situações, a participação da tiamina na patogênese da PEM pode ser questionada, uma vez que não há alterações nas concentrações sanguíneas dessa substância.

Para o estudo da patogênese da PEM dos ruminantes, é indicado o estabelecimento de um modelo experimental do qual se conheçam detalhadamente a dose do agente causador, a evolução dos sinais clínicos, o tipo e a distribuição das lesões no sistema nervoso central. Embora existam trabalhos que mencionam alguns aspectos neuropatológicos da intoxicação por amprólio em ruminantes (Loew \& Dunlop, 1972, Markson et al. 1974, Morgan et al. 1975, Kasahara et al. 1989), pouco se conhece a respeito da caracterização morfológica das alterações produzidas por esse antagonista da tiamina, bem como sobre a distribuição dessas lesões no encéfalo.

O objetivo desse trabalho é descrever os achados clinicopatológicos e a distribuição das alterações no encé- falo em ovinos intoxicados experimentalmente com amprólio.

\section{MATERIAL E MÉTODOS}

Foram utilizados cinco ovinos mestiços, fêmeas, de seis meses de idade, com peso entre 18-29 kg que foram everminados e numerados de 1-6 antes do início do experimento (Quadro 1). Durante o período de adaptação de 15 dias e por todo o experimento os ovinos foram mantidos em baias descobertas de $5 \times 2 \mathrm{~m}$ e foram pesados ao início do experimento e a cada 15 dias. Diariamente era realizado exame físico visando principalmente a detecção de sinais clínicos neurológicos. Os ovinos receberam água a vontade e $2 \%$ do peso vivo em matéria seca na forma de feno de alfafa e ração comercial para ovinos.

Cinco ovinos receberam amprólio (Amprolbase [40\% de amprólio]) ${ }^{6}$ nas dosagens de $500 \mathrm{mg} / \mathrm{kg} / \mathrm{dia}$ (Grupo 1, Ovinos 1 e 2) e $1.000 \mathrm{mg} / \mathrm{kg} / \mathrm{dia}$ (Grupo 2, Ovinos 3-5) até a manifestação dos primeiros sinais clínicos neurológicos. O amprólio era pesado diariamente em balança de precisão e acondicionado em sacos plásticos individuais que eram identificados com o número do ovino que receberia aquela porção. A administração do produto era feita por via oral, através de ingestão forçada com uma seringa de $20 \mathrm{~mL}$, da qual foi cortada a parte anterior para facilitar a administração. Os ovinos morreram naturalmente (Ovinos 1, 4 e 5) ou foram submetidos à eutanásia in extremis (Ovinos 2 e 3). Todos os ovinos foram necropsiados.

O encéfalo juntamente com o complexo gânglio de Gasser, rete mirabile carotídea e hipófise $(\mathrm{GRH})$, e fragmentos de diversos outros órgãos eram coletados e fixados em formol a $10 \%$. Após a fixação, eram realizados cortes transversais no encéfalo com intervalos de $1 \mathrm{~cm}$ para avaliação macroscópica. As seguintes seções eram preparadas para avaliação microscópica: cerebelo, bulbo na altura do óbex, ponte com pedúnculos cerebelares, mesencéfalo na altura dos colículos rostrais, tálamo, núcleos basais, hipocampo e lobos frontal, parietal e occipital do telencéfalo. Adicionalmente, o exame histológico era realizado em um monobloco do complexo GRH. Todos os fragmentos eram processados rotineiramente para histologia, incluídos em parafina e corados pela hematoxilina-eosina.

As alterações observadas no encéfalo foram graduadas de acordo com a intensidade numa escala de 0 (ausência de lesão) a 3 (lesão acentuada). Cada lesão tinha seu grau aferido de acordo com a média de percepção subjetiva de três patologistas que examinaram todos os casos. Foram avaliados o grau de intensidade e a distribuição nos diferentes locais examinados procurando-se pelas seguintes alterações histológicas: gliose focal, gliose difusa, tumefação de núcleos de células endoteliais, separação entre as substâncias cinzenta e branca, edema do neurópilo, congestão, hemorragia, necrose neuronal (caracterizada por picnose nuclear e eosinofilia e encarquilhamento citoplasmáticos), neuronofagia, malacia (o termo malacia foi empregado aqui significando necrose de todas as células neuroectodérmicas com manutenção das células mesenquimais (i.é, células gitter, células endoteliais) que sobrevivem e participam do processo (Innes \& Saunders 1962), lesão residual (estrutura cavitária formada por pequenos vasos e numerosas células gitter remanescen-

\footnotetext{
${ }^{6}$ Amprolbase, Laboratório Farmabase Saúde Animal, Av. Emílio Marconato 1000, Galpão A3, Chácara Primavera, Jaguariúna, SP 13820-000. www.farmabase.com.br
} 
tes) e infiltrado inflamatório perivascular e/ou meníngeo. A distribuição do edema e da necrose neuronal (neurônio vermelho) nas lâminas da substância cinzenta do telencéfalo era também determinada.

\section{RESULTADOS}

Dos cinco ovinos que receberam amprólio, três morreram espontaneamente (Ovinos 1, 4 e 5) e outros dois foram eutanasiados in extremis. Os primeiros sinais clínicos surgiram 28-59 e 13-39 dias após a administração do amprólio nas dosagens de 500 e $1.000 \mathrm{mg} / \mathrm{kg}$, respectivamente (Quadro 1). A evolução clínica variou de 3-7 dias. Os sinais clínicos neurológicos eram consistentes em todos os casos e compreendiam prostração, incoordenação, midríase, bruxismo, cegueira, decúbito lateral, movimentos de pedalagem e opistótono (Fig.1). Adicionalmente, um ovino manifestou sialorréia e assumiu posição de cavalete e outro apresentou mioclonias.

Macroscopicamente, as principais alterações eram restritas ao sistema nervoso central (Quadro 1) e incluíam tumefação do encéfalo com achatamento das circunvoluções (Fig.2) nos Ovinos 1, 2, 4 e 5; áreas de hemorragia nos lobos parietal e occipital nos Ovinos 2 e 3 , na região submeníngea da medula espinhal cervical dos Ovinos 3 e 4 e no mesencéfalo do Ovino 2 (Fig.3). Hemorragias eram também observadas no coração dos Ovinos 1 e 5 e no gânglio de Gasser do Ovino 1 e no

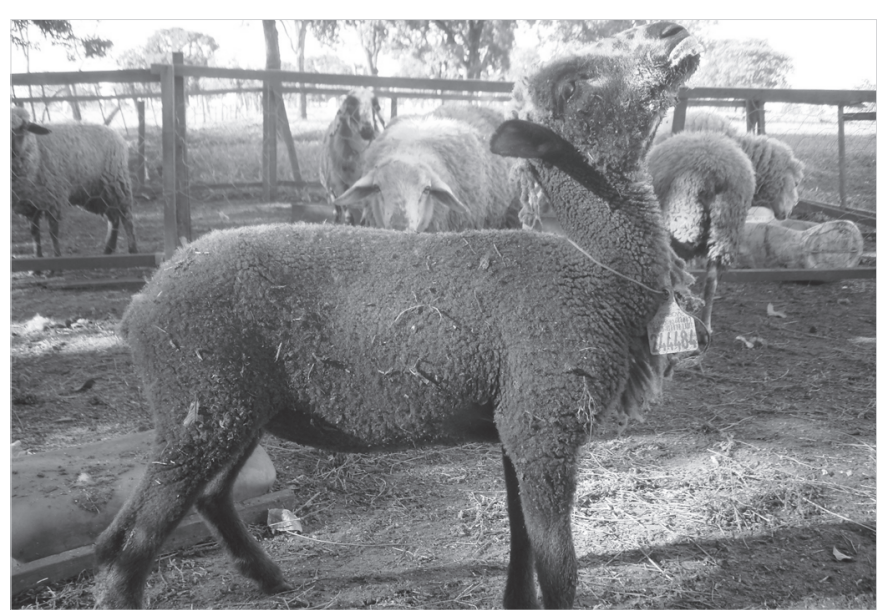

Fig.1. Ovino 3 em opistótono na polioencefalomalacia induzida por amprólio.

fígado do Ovino 4; congestão e edema pulmonar acentuados ocorreram no Ovino 1.

Lesões microscópicas incluíam necrose neuronal (neurônios vermelhos), segmentar e laminar, acompanhada de edema (Fig.4), tumefação de núcleos das células endoteliais (Fig.5), hemorragia (Fig.6) e infiltrado de macrófagos espumosos (células gitter) (Fig.7). Essas alterações foram mais acentuadas nos lobos frontal, parietal e occipital; o limite entre as lesões e a área adjacente nor-

\section{Quadro 1. Polioencefalomalacia em ovinos intoxicados experimentalmente por amprólio}

\begin{tabular}{|c|c|c|c|c|c|c|}
\hline Ovino & $\begin{array}{c}\text { Peso } \\
(\mathrm{kg})\end{array}$ & $\begin{array}{l}\text { Quantidade } \\
\text { de amprólio } \\
\text { administrado } \\
\text { (mg/kg/dia) }\end{array}$ & $\begin{array}{l}\text { Período de } \\
\text { administra- } \\
\text { ção (dias) }\end{array}$ & $\begin{array}{l}\text { Duração dos } \\
\text { sinais clíni- } \\
\text { cos (dias) }\end{array}$ & Sinais clínicos neurológicos & Achados de necropsia \\
\hline 1 & 28 & 500 & 28 & 7 & $\begin{array}{l}\text { Cegueira, prostração, bruxismo, incoordena- } \\
\text { nação, decúbito lateral, opistótono, midríase } \\
\text { e movimentos de pedalagem. Morte espontâ- } \\
\text { nea. }\end{array}$ & $\begin{array}{l}\text { Congestão e edema pulmonar acentua- } \\
\text { dos, hemorragias no coração (endocár- } \\
\text { dio, miocárdio e epicárdio), hemorragia } \\
\text { no gânglio de Gasser, tumefação do en- } \\
\text { céfalo e achatamento das circunvolu- } \\
\text { ções cerebrais. }\end{array}$ \\
\hline 2 & 27 & 500 & 59 & 6 & $\begin{array}{l}\text { Prostração, incoordenação, opistótono em } \\
\text { estação, decúbito lateral, midríase, hiperexci- } \\
\text { tabilidade e bruxismo. Submetido à eutanásia. }\end{array}$ & $\begin{array}{l}\text { Achatamento das circunvoluções cere- } \\
\text { brais, superfície natural do telencéfalo } \\
\text { com áreas multiocais amarelas; superfí- } \\
\text { cie de corte do mesencéfalo e do telen- } \\
\text { céfalos occipital e parietal com petéquias } \\
\text { e equimoses multifocais. }\end{array}$ \\
\hline 3 & 23 & 1.000 & 13 & 3 & $\begin{array}{l}\text { Isolamento do grupo, prostração, decúbito } \\
\text { esternal, incoordenação, decúbito lateral, opis- } \\
\text { tótono, nistagmo, cegueira, paralisia de mem- } \\
\text { bros torácicos e pélvicos, estrabismo, midría- } \\
\text { se, movimentos de pedalagem. Submetido à } \\
\text { eutanásia. }\end{array}$ & $\begin{array}{l}\text { Hemorragia submeníngea nos lobos pa- } \\
\text { rietal e occipital esquerdos e na medula } \\
\text { espinhal cervical. }\end{array}$ \\
\hline 4 & 20 & 1.000 & 20 & 4 & $\begin{array}{l}\text { Depressão, incoordenação, cegueira, nistag- } \\
\text { mo, decúbito lateral, movimentos de pedala- } \\
\text { gem, opistótono. Morte espontânea. }\end{array}$ & $\begin{array}{l}\text { Palidez de mucosas, petéquias no lobo } \\
\text { acessório do fígado, tumefação do encé- } \\
\text { falo, achatamento das circunvoluções } \\
\text { cerebrais e hemorragias submeníngeas } \\
\text { na medula espinhal cervical. }\end{array}$ \\
\hline 5 & 18 & 1.000 & 39 & 4 & $\begin{array}{l}\text { Letargia, mioclonias, incoordenação, opistóto- } \\
\text { no em estação, sialorréia, bruxismo, posição } \\
\text { de cavalete, decúbito lateral, cegueira, respi- } \\
\text { ração abdominal, movimentos de pedalagem. } \\
\text { Morte espontânea. }\end{array}$ & $\begin{array}{l}\text { Petéquias no epicárdio, tumefação do } \\
\text { encéfalo e achatamento das circunvolu- } \\
\text { ções cerebrais. }\end{array}$ \\
\hline
\end{tabular}



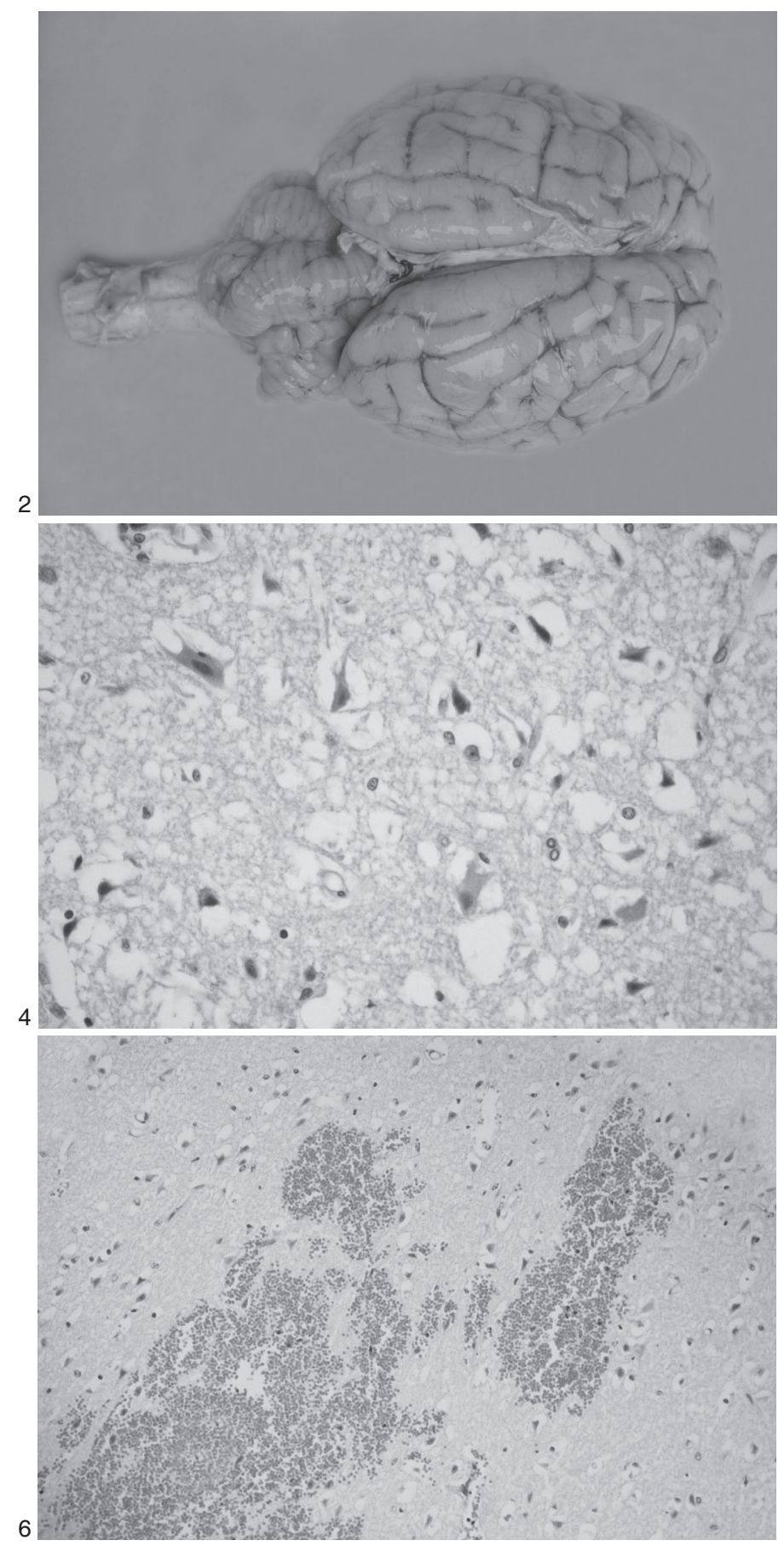

Fig.2. Encéfalo do Ovino $1 \mathrm{com}$ polioencefalomalacia induzida por amprólio. O encéfalo está tumefeito e há achatamento das circunvoluções.

Fig.4. Córtex parietal do Ovino 3 com polioencefalomalacia induzida por amprólio. Há necrose de neurônios que têm o citoplasma acidofílico e núcleo picnótico (neurônios vermeIhos). O neurópilo é frouxo e vacuolizado (edema). HE, obj.40x.

Fig.6. Áreas de hemorragia no córtex parietal do Ovino 3 com polioencefalomalacia induzida por amprólio. HE, obj.20x.
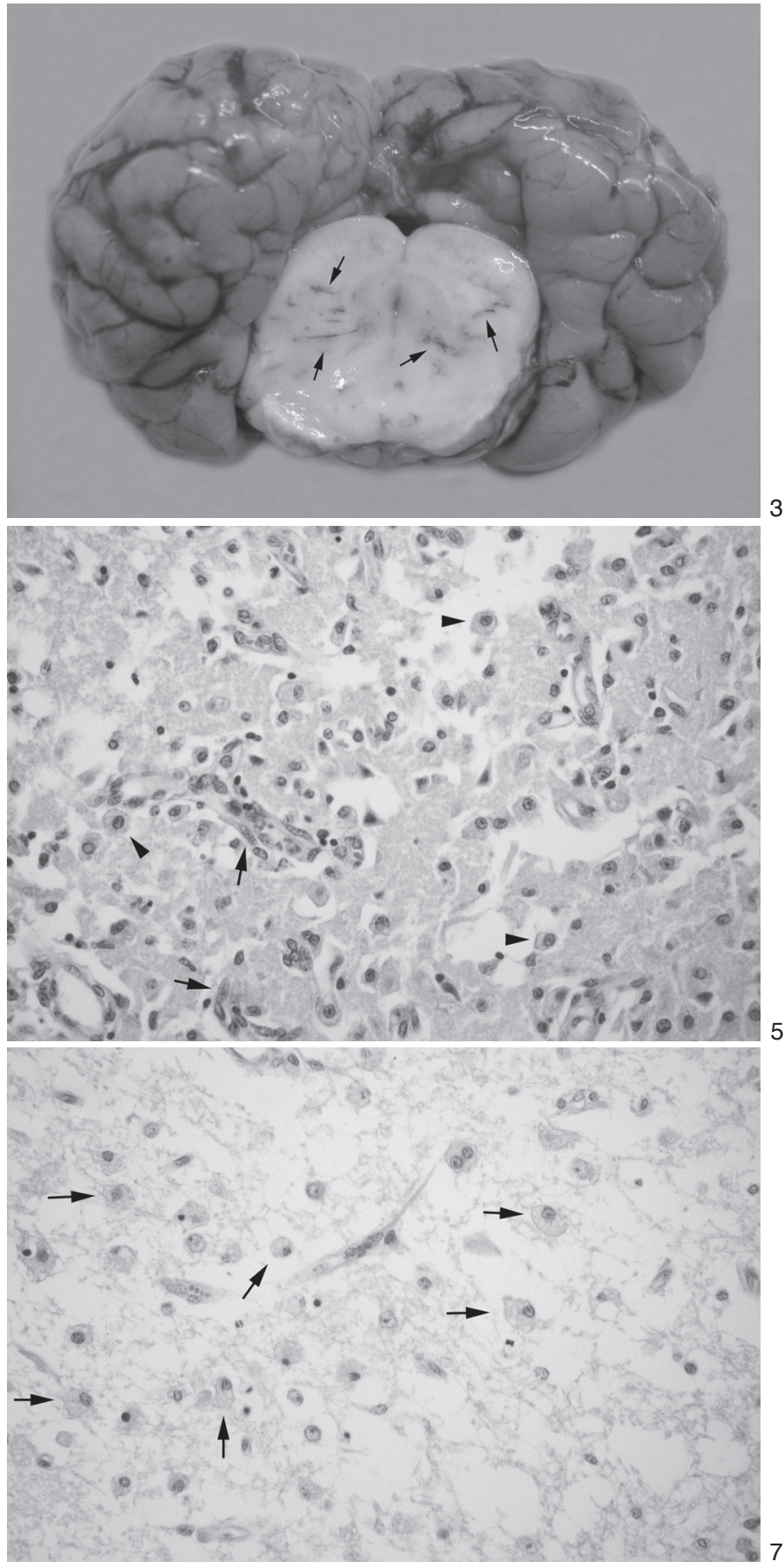

Fig.3. Encéfalo do Ovino 2 com polioencefalomalacia induzida por amprólio. Há hemorragias simétricas (setas) no mesencéfalo.

Fig.5. Córtex frontal do Ovino $1 \mathrm{com}$ polioencefalomalacia induzida por amprólio. Há tumefação das células endoteliais dos vasos (setas), neurônios necróticos (neurônios vermeIhos), edema e células gitter (cabeças de seta). HE, obj.40x.

Fig.7. Macrófagos espumosos (células gitter) (setas) no teto do mesencéfalo do Ovino $1 \mathrm{com}$ polioencefalomalacia induzida por amprólio. HE, obj.40x. 


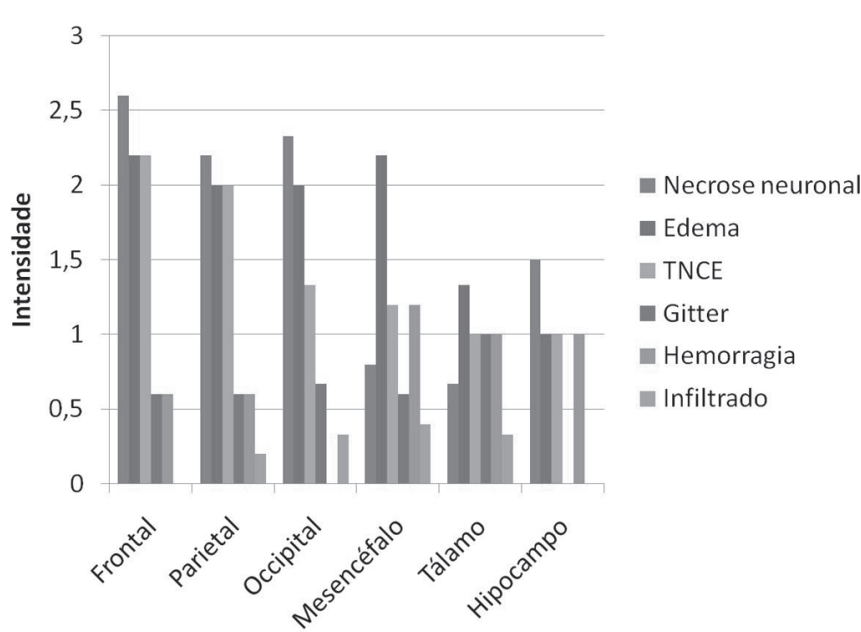

Fig.8. Tipo, distribuição e intensidade das lesões induzidas experimentalmente por amprólio no encéfalo de cinco ovinos. Os números que aparecem na ordenada referem-se a intensidade da lesão: $0=$ ausência de lesão 1 = lesão leve; 2 = moderada; 3 = acentuada. $\mathrm{Na}$ abscissa estão os locais do cérebro (lobos frontal, parietal, occipital; mesencéfalo, tálamo, hipocampo) onde ocorriam as lesões.

mal era abrupto. Adicionalmente, lesões similares, moderadas ou acentuadas, mas com menor intensidade de necrose neuronal, eram encontradas no mesencéfalo, tálamo e hipocampo. A Figura 8 mostra a distribuição e intensidade dessas lesões. Em nenhum ovino foram observadas alterações histológicas no bulbo, na ponte e no complexo GRH. Apenas um ovino apresentou grau leve de tumefação de núcleos das células endoteliais nos núcleos basais. Não houve diferença de intensidade de lesões macro e microscópicas entre os dois grupos avaliados. Os neurônios necróticos eram caracterizados por encarquilhamento e hipereosinofilia do citoplasma, picnose nuclear sem evidenciação nucleolar e desaparecimento da substância de Nissl. Alguns núcleos de neurônios necróticos apresentavam aspecto vítreo. A necrose neuronal ocorreu tanto no topo quanto no sulco das circunvoluções cerebrais. $O$ edema na substância cinzenta era acentuado e, em alguns casos, deixava o neurópilo com aspecto esponjoso; ocorria separando a substância cinzenta da branca ou entre camadas de neurônios da substância cinzenta. Em dois ovinos, havia manguitos perivasculares focais leves de linfócitos e macrófagos. Hemorragias multifocais e moderadas eram encontradas com frequência nas áreas de malacia nos telencéfalos, bem como no mesencéfalo, tálamo e hipocampo. Em nenhum caso foi observada neuronofagia nem lesão residual (cicatriz). Lesões no tronco encefálico ocorreram em todos os ovinos e eram leves ou moderadas e restritas ao teto do mesencéfalo; caracterizavam-se por áreas geralmente simétricas e bilaterais de necrose neuronal focal, edema do neurópilo, tumefação de núcleos de células endoteliais, hemorragias moderadas e ocasionais células gitter. No tálamo, as alterações eram semelhantes às do mesencéfalo, porém o edema era menos acentuado. A distribuição da necrose neuronal e do edema nas camadas de neurônios da substância cinzenta dos lobos fron- tal, parietal e occipital do telencéfalo, não diferiu entre os ovinos, afetando uniformemente todas as camadas.

\section{DISCUSSÃO}

No presente estudo foi constatado que a dosagem diária de $500 \mathrm{mg} / \mathrm{kg}$ de amprólio é eficaz no desenvolvimento de $P E M$ em ovinos e, que na dosagem de $1.000 \mathrm{mg} / \mathrm{kg} \mathrm{a}$ doença ocorre precocemente, visto que o ovino 3 adoeceu no $13^{\circ}$ dia de experimento. Entretanto, o tipo e a intensidade das lesões histológicas observadas nos dois grupos foram idênticas. Em outro trabalho com administração de $880 \mathrm{mg} / \mathrm{kg}$ de amprólio à ovinos adultos, a doença foi reproduzida e os primeiros sinais clínicos surgiram com 37 dias (Loew \& Dunlop 1972). Bezerros que receberam dieta com $1 \%$ de amprólio desenvolveram PEM com 25-39 dias de experimentação (Markson et al. 1974).

Os sinais clínicos apresentados pelos ovinos deste estudo estão associados às lesões corticais e ao edema que causou compressão do tronco encefálico e de outras estruturas ventrais do encéfalo e foram semelhantes aos relatados em outras descrições de PEM associada às intoxicações por amprólio (Loew \& Dunlop 1972) e por enxofre (Rousseaux et al. 1991, McAllister et al. 1992, Jeffrey et al. 1994) em ovinos. Bradicardia e inatividade do rúmen também são descritas em alguns ovinos afetados pela doença (Loew \& Dunlop 1972). Cordeiros pré-ruminantes intoxicados diariamente com $280 \mathrm{mg} / \mathrm{kg}$ apresentaram diarreia mucoide esverdeada e epistaxe (Morgan et al. 1975). Adicionalmente, anorexia, diarreia, andar rígido, miose, espasmos clônicos e hipotermia foram observados em bezerros intoxicados experimentalmente por amprólio (Markson et al. 1974, Kasahara et al. 1989).

As alterações macroscópicas do encéfalo observadas neste estudo são semelhantes às descritas em outros trabalhos de PEM em ovinos (Morgan et al. 1975, Rousseaux et al. 1991). Em alguns casos de PEM nessa espécie, não são observadas lesões macroscópicas (Vieira et al. 2007). Áreas de hemorragia no encéfalo são relatadas em PEM associada às intoxicações por amprólio (Morgan 1974, Morgan et al. 1975) ou, ocasionalmente, na toxicose por enxofre (Hamlen et al. 1993). Essa alteração parece ser importante nesse tipo de PEM causada por excesso de amprólio, uma vez que não ocorre com frequência na doença associada a outras causas.

Embora as lesões histológicas tenham sido mais intensas nos lobos frontal, parietal e occipital, alterações moderadas a acentuadas também foram detectadas no tálamo, mesencéfalo e hipocampo. Esse tipo de lesão cortical e de estruturas mais ventrais do encéfalo com hemorragias é considerada uma forma grave da doença e tem sido observada na PEM em bovinos do Brasil (Sant'Ana et al. 2009) e de outros países associada ao consumo excessivo de enxofre (Hamlen et al. 1993, Hill \& Ebbett 1997, Gould 2000). Entretanto, nesses casos causados por enxofre, há degeneração de veias e vênulas (Loneragan et al. 1998) e necrose fibrinóide de pequenas arteríolas no tálamo (Hamlen et al. 1993) e mesencéfalo 
(Hamlen et al. 1993, Hill \& Ebbett 1997), o que não foi observado na intoxicação por amprólio do presente estudo. Nos telencéfalos de casos experimentais deste trabaIho, o limite entre a substância cinzenta afetada e a íntegra é abrupto, diferente do observado em casos naturais de PEM em bovinos (Sant'Ana et al. 2009). Não foram verificadas lesões no cerebelo dos ovinos. Em bovinos, alterações nessa estrutura geralmente ocorrem em função de pressão exercida durante a herniação pelo forame magno (Morgan 1974, Sant'Ana et al. 2009).

A necrose neuronal e o edema ocorreram homogeneamente entre as lâminas de neurônios do córtex telencefálico, semelhantemente ao observado em ovinos intoxicados experimentalmente por amprólio (Morgan 1974) e naturalmente por enxofre (Bulgin et al. 1996, Low et al. 1996). Em casos espontâneos em bovinos, as camadas granular externa e interna do córtex occipital, parietal e frontal foram mais afetadas por necrose de neurônios e edema (Sant'Ana et al. 2009). Na PEM associada à intoxicação por enxofre em bovinos, a necrose predomina nas camadas corticais superiores e médias de neurônios piramidais (Hamlen et al. 1993, Jeffrey et al. 1994) e a espongiose ocorre especialmente nas camadas profundas (Gould 2000).

O conhecimento das doses de amprólio necessárias para o estabelecimento de PEM em ovinos, a determinação dos sinais clínicos, da evolução da doença, do tipo e da distribuição das lesões no sistema nervoso central, permite estabelecer um modelo experimental em ovinos para o estudo da PEM em ruminantes.

\section{REFERÊNCIAS}

Anderton N., Cockrum P.A., Walker D.W. \& Edgar J.A. 1994. Identification of a toxin suspected of causing death in livestock grazing Phalaris pastures, p.269-274. In: Colegate S.M. \& Dorling P.R. (Eds), Plant-Associated Toxins. Agricultural, Phytochemical and Ecological Aspects. CAB International, Farnham Royal, Slough, UK,.596p.

Bulgin M.S., Lincoln S.D. \& Mather G. 1996. Elemental sulfur toxicosis in a flock of sheep. J. Am. Vet. Med. Assoc. 208:1063-1065.

Carrillo B.J., Pospischil A. \& Dahme E. 1983. Pathology of a bovine necrotizing encephalitis in Argentina. Zentralbl. Veterinärmed. Reihe B 30:161-168.

Gould D.H. 2000. Update on sulfur-related polioencephalomalacia. Vet. Clin. North Am., Food Anim.Pract. 16:481-496.

Hamlen H., Clark E. \& Janzen E. 1993. Polioencephalomalacia in cattle consuming water with elevated sodium sulfate levels: A herd investigation. Can. Vet. J. 34:153-158.

Hill F.I. \& Ebbett P.C. 1997. Polioencephalomalacia in cattle in New Zealand fed chou moellier (Brassica oleracea). N. Z. Vet. J. 45:37-39.

Innes J.R.M. \& Saunders L.Z. 1962. Encephalomalacia and myelomalacia, p.607-609. In: Ibid. (Eds), Comparative Neuropathology. Academic Press, New York. 839p.

Jeffrey M., Duff J.P., Higgins R.J., Simpson V.R., Jackman R., Jones
T.O., Mechie S.C. \& Liversey C.T. 1994. Polioencephalomalacia associated with the ingestion of ammonium sulphate by sheep and cattle. Vet. Rec. 134:343-348.

Jensen R., Griner L.A. \& Adams O.R. 1956. Polioencephalomalacia of cattle and sheep. J. Am. Vet. Med. Assoc. 129:311-321.

Kasahara T., Ichijo S., Osame S. \& Sarashina T. 1989. Clinical and biochemical findings in bovine cerebrocortical necrosis produced by oral administration of amprolium. Jpn. J. Vet. Sci. 51:79-85.

Lemos R.A.A., Driemeier D., Guimarães E.B., Dutra I.S., Mori A.E. \& Barros C.S.L. 2004. Lead poisoning in cattle grazing pasture contaminated by industrial waste. Vet. Human Toxicol. 46:326-328.

Lindley W.H. 1977. Water deprivation in cattle. J. Am. Vet. Med. Assoc. 171:439-440.

Linklater K.A., Dyson D.A. \& Morgan K.T. 1977. Faecal thiaminase in clinically normal sheep associated with outbreaks of polioencephalomalacia. Res. Vet. Sci. 22:308-312.

Loew F.M. \& Dunlop R.H. 1972. Induction of thiamine inadequacy and polioencephalomalacia in adult sheep with amprolium. Am. J. Vet. Res. 33:2195-2205.

Loneragan G.H., Gould D.H., Callan R.J., Sigurdson C.J. \& Hamar D.W. 1998. Association of excess sulfur intake and an increase in hydrogen sulfide concentrations in the ruminal gas cap of recently weaned beef calves with polioencephalomalacia. J. Am. Vet. Med. Assoc. 213:15991604.

Low J.C., Scott P.R., Howie F., Lewis M., FitzSimons J. \& Spence J.A. 1996. Sulphur-induced polioencephalomalacia in lambs. Vet. Rec. 138:327-329.

McAllister M.M., Gould D.H. \& Hamar D.W. 1992. Sulphide-induced polioencephalomalacia in lambs. J. Comp. Path. 106:267-278.

Markson L.M., Edwin E.E., Lewis G. \& Richardson C. 1974. The production of cerebrocortical necrosis in ruminant calves by the intraruminal administration of amprolium. Brit. Vet. J. 130:9-16.

Mella C.M., Perez-Oliva O. \& Loew F.M. 1976. Induction of bovine polioencephalomalacia with a feeding system based on molasses and urea. Can. J. Comp. Med. 40:104-110.

Morgan K.T. 1974. Amprolium poisoning of preruminant lambs: An ultrastructural study of the cerebral malacia and the nature of the inflammatory response. J. Path. 112:229-236

Morgan K.T., Coop R.L. \& Doxey D.L. 1975. Amprolium poisoning of preruminant lambs. An investigation of the encephalopathy and the haemorrhagic and diarrhoeic syndromes. J. Path. 116:73-81.

Moro L., Nogueira R.H.G., Carvalho A.U. \& Marques D.C. 1994. Relato de três casos de polioencefalomalácia em bovinos. Arq. Bras. Med. Vet. Zootec. 46:409-416.

Ramos J.J., Ferrer L.M., García L., Fernández A. \& Loste A. 2005. Polioencephalomalacia in adult sheep grazing pastures with prostate pigweed. Can. Vet. J. 46:59-61.

Rousseaux C.G., Olkowski A.A., Chauvet A., Gooneratne S.R. \& Christenson D.A. 1991. Ovine polioencephalomalacia associated with dietary sulphur intake. J. Vet. Med. A 38:229-239.

Sant'Ana F.J.F., Rissi D.R., Lucena R.B., Lemos R.A.A., Nogueira A.P.A. \& Barros C.S.L. 2009. Polioencefalomalácia em bovinos: epidemiologia, sinais clínicos e distribuição das lesões no encéfalo. Pesq. Vet. Bras. 29:487-497.

Vieira A.C.S., Afonso J.A.B., Tokarnia C.H., Costa N.A., Mendonça C.L. \& Souza M.I. 2007. Estudo epidemiológico, clínico e patológico em ruminantes com polioencefalomalácia no agreste meridional de Pernambuco. Revta Bras. Med. Vet. 29:65-68. 